\title{
Two Rhinoliths in a Single Nasal Cavity in an Elderly Patient Mimicking Fungal Sinusitis
}

\author{
${ }^{1}$ Vishal Prasad, ${ }^{2}$ Chandrakala Srinivas, ${ }^{3}$ Sheetal Krishnappa, ${ }^{4}$ Suresh T Narayan Rao, ${ }^{5}$ Harshitha T Rajanna
}

\begin{abstract}
Objective: To highlight an interesting and a rare case of two rhinoliths in a single nasal cavity of an elderly male patient, mimicking fungal sinusitis or malignancy.

To emphasize that rhinolith has to be considered in the differential diagnosis of unilateral nasal mass even in the elderly.

Case report: A 55-year-old man presented with a right-sided nasal obstruction, foul-smelling nasal discharge and a mass in the right nasal cavity of 6 months duration. Endoscopic examination revealed a gritty, blackish brown mass filling the entire right nasal cavity. A clinical differential diagnosis of malignancy or fungal sinusitis was made. CT scan of paranasal sinus was suggestive of fungal sinusitis following which patient was posted for biopsy. Intraoperatively, patient was found to have two separate masses in the right nasal cavity which were removed. We found a septal perforation adjoining the area where the rhinolith was impacted, with septum pushed laterally and adherent to middle turbinate. However, there were no features suggestive of malignancy. Histopathological examination also ruled out malignancy and confirmed a vegetable foreign body with calcification around an acellular material.
\end{abstract}

Conclusion: Rhinolith, though rare should be considered in the differential diagnosis of unilateral nasal mass in the elderly.

Keywords: Rhinolith, Septal perforation, Nasal mass, Fungal sinusitis.

How to cite this article: Prasad V, Srinivas C, Krishnappa S, Rao STN, Rajanna HT. Two Rhinoliths in a Single Nasal Cavity in an Elderly Patient Mimicking Fungal Sinusitis. Clin Rhinol An Int J 2014;7(2):76-79.

\section{Source of support: Nil}

Conflict of interest: None declared

\section{INTRODUCTION}

Rhinolith is a rare entity in elderly and to have two rhinoliths in a single nasal cavity is still rarer. ${ }^{1,2}$ In literature there are

\footnotetext{
${ }^{1}$ Lecturer, ${ }^{2,4}$ Associate Professor, ${ }^{3,5}$ Junior Resident

${ }^{1-3,5}$ Department of ENT, Sri Devaraj Urs Medical College Kolar, Karnataka, India

${ }^{4}$ Department of Pathology, Sri Devaraj Urs Medical College Kolar, Karnataka, India
}

Corresponding Author: Vishal Prasad, Lecturer, Department of ENT, Sri Devaraj Urs Medical College, Kolar, Karnataka, India Phone: 8105185861, e-mail: drvishal_prasad@yahoo.co.in only about 600 reported cases of rhinoliths. Diagnosis of rhinolith is often overlooked. They are often diagnosed accidently, on routine examination or while treating for other associated conditions like sinusitis. We highlight a rare case of two rhinoliths seen in an elderly individual with a black necrotic mass protruding through nasal cavity, mimicking fungal sinusitis or malignancy.

\section{CASE REPORT}

A 55-year-old man presented to the Department of Otorhinolaryngology with a right-sided nasal obstruction and foulsmelling nasal discharge since 1 year with a mass protruding from right nasal cavity and facial pain of 6 months duration. There was no history of foreign body insertion into nose in the past. The patient was initially evaluated at a tertiary center and was suspected to have fungal sinusitis.

Anterior rhinoscopic examination revealed a gritty, blackish brown mass protruding into nasal cavity and filling the entire right nasal cavity. The mass could be probed on all sides and did not bleed on touch. Endoscopic examination was performed on the left side only as the mass had filled the right nasal cavity. The septum had two perforations and was pushed to left, almost touching the lateral wall. Nasopharynx was visualized by passing a $30^{\circ}$ scope through left nasal cavity and was found normal. Examination of oral cavity and oropharynx showed no abnormality. Axial/ coronal computed tomography of nose and paranasal sinuses revealed a soft-tissue mass within the right maxillary sinus which extended to the right nasal cavity, ethmoid and frontal sinus. A clump of large coarse calcification measuring $2.4 \times$ $2.4 \times 1.5 \mathrm{~cm}$ was present within the mass (Fig. 1). There was significant expansion and sclerosis of the walls of the ethmoid bone with architectural distortion of sinus anatomy.

A differential diagnosis of sinonasal malignancy or fungal rhinosinusitis was made based on clinical and radiological findings.

Patient was taken up for biopsy to confirm the diagnosis. An informed written consent was taken both for the procedure as well as publication. On table, we found two separate masses in the right nasal cavity which were blackish brown and hard in consistency and were removed (Fig. 2).

After removal of the mass, examination showed wide and roomy right nasal cavity, septum was perforated at two 

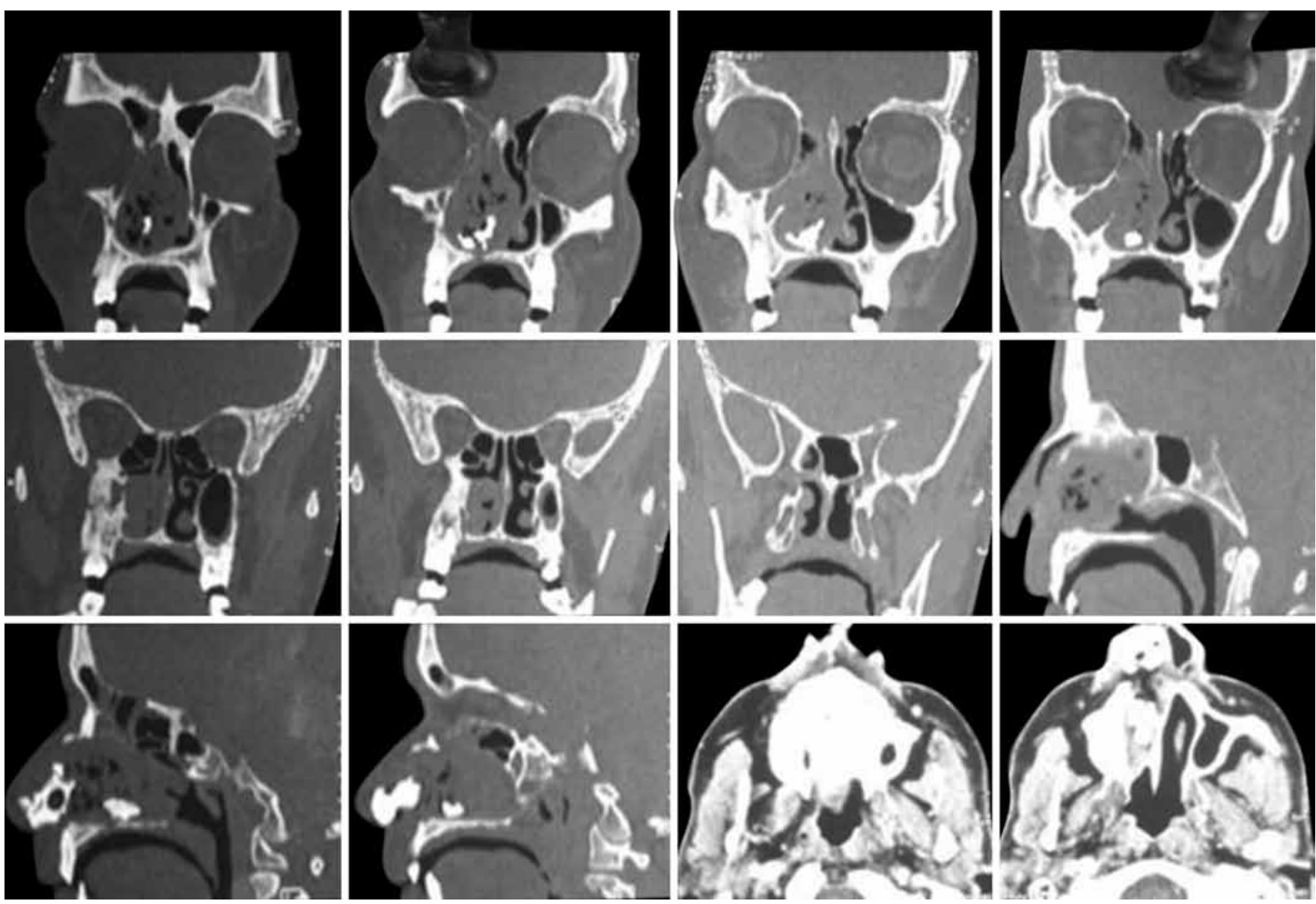

Fig. 1: Noncontrast CT paranasal sinusitis showing soft-tissue mass in the right maxillary, ethmoid and frontal sinus with an area of coarse calcification

sites adjoining the area where the rhinolith was impacted and pushed to the left due to mass effect (Fig. 3). The right middle turbinate was lateralized and was attached to uncinate process leading to blockage of osteomeatal complex. However, nasal mucosa appeared normal and there were no features suggestive of malignancy. A diagnosis of rhinolith with adjoining granulation tissue was made and the specimen was sent for histopathological confirmation.

Histopathological examination revealed a mass having a central vegetable foreign body with surrounding acellular material and calcifications confirming the diagnosis (Fig. 4).

Patient underwent endoscopic sinus surgery to treat the sinusitis that could have been secondary to osteomeatal complex obstruction by the rhinolith. Right frontal, maxillary and ethmoid sinuses were cleared of the disease. Postoperative course was uneventful and patient was discharged on 4th postoperative day.

\section{DISCUSSION}

Foreign body in the nose is a common entity, but the incidence of rhinolith is very rare in the elderly. Rhinolith was first described by Bartholini in $1654 .^{2,3}$ It is reported that less than $0.01 \%$ patients present with rhinolith. ${ }^{4}$ There are around 600 cases of rhinolith that have been reported till date. They are unilateral, single and vary widely in size and shape. ${ }^{4}$ Here we report a rare case of two rhinoliths in a single nasal cavity presenting at an elderly age. One study quotes that rhinoliths occur in all age groups and mostly found in lower socioeconomic status. ${ }^{5}$ However, few other studies have reported that the age group frequently affected is between 8 and 25 years. ${ }^{6-8}$ It is seen that Rhinolith occurs more common in females. ${ }^{4,5}$ Common sites in the nasal cavity are on the floor, between the maxillary sinus wall and inferior turbinate or between the inferior turbinate and septum. ${ }^{5,9}$ In our case, the patient was an elderly male with two rhinoliths in a single nasal cavity, in contrast to the other reports.

Rhinolith occurs as a result of calcareous concretions, around a long standing intranasal foreign body. The calcified foreign bodies in the nose were formerly designated false or true rhinoliths. These terms have been replaced by exogenous and endogenous, depending on whether or not a nucleus, around which the incrustation has been deposited. ${ }^{3}$ Rhinoliths that have developed around materials introduced into the nose such as cherry stones, forgotten nasal swabs, vegetative matter and other articles like sand, bead, paper, buttons, wood or glass are called exogenous. Endogenous rhinoliths are those that are formed around body's own 


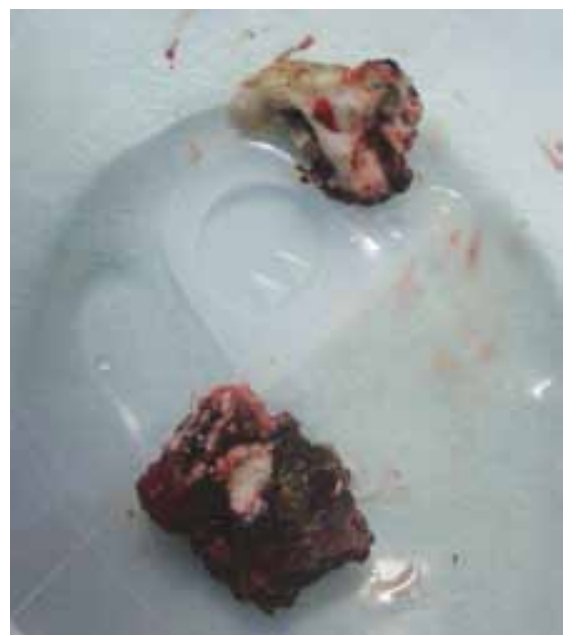

Fig. 2: Two excised specimen from right nasal cavity sent for histopathology

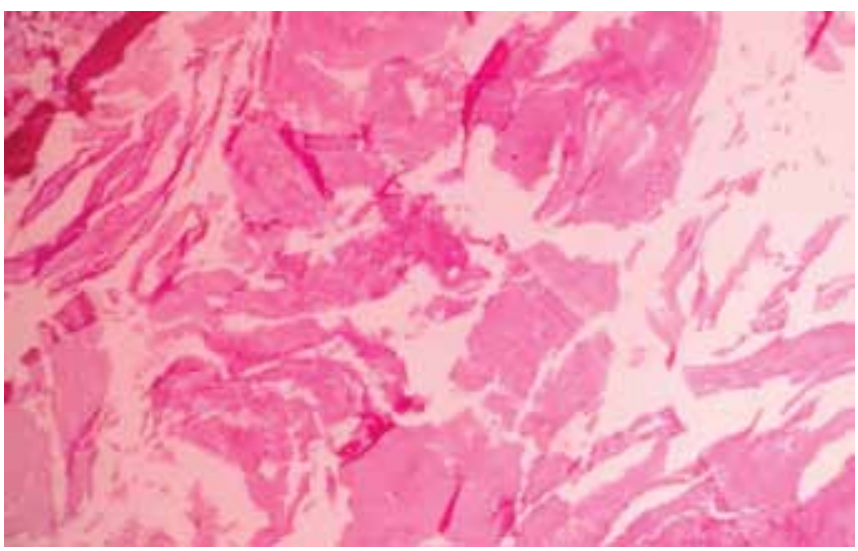

Fig. 4: Histopathological examination showing a mass with central vegetable foreign body with surrounding acellular material and calcifications ( $\mathrm{H}$ and $\mathrm{E}$ stain, $\times 100)$

material such as ectopic teeth in the maxillary sinus, bone sequestra, dried blood clots, desquamated epithelium, dried pus and inspissated mucus in the nasal cavity. ${ }^{2,9,10}$ The chemical composition of rhinolith is hydroxy apatite, calcium phosphate hydrate, magnesium phosphate, carbonate and oxalate ions. Morgan described that in an acidic $\mathrm{pH}$, oxalates were deposited around the nidus leading to hard stone formation. Some studies have reported phosphates deposition in alkaline medium forming soft and friable stones. ${ }^{10}$ Nover and Florke reported that they are chiefly composed of siderite and ferrihydrite with nucleus of high iron content rather than of calcium or magnesium salts. ${ }^{11}$

Diagnosis of the rhinolith is often overlooked. Most of the patients, including our patient do not recall the history of foreign body insertion into their nose. They present with the symptoms dating back to several months or years as seen in our case. Patients often presents with foul smelling, purulent rhinorrhea, unilateral nasal obstruction, headache, facial pain, anosmia and fever. ${ }^{4,9,11}$ Our patient presented with

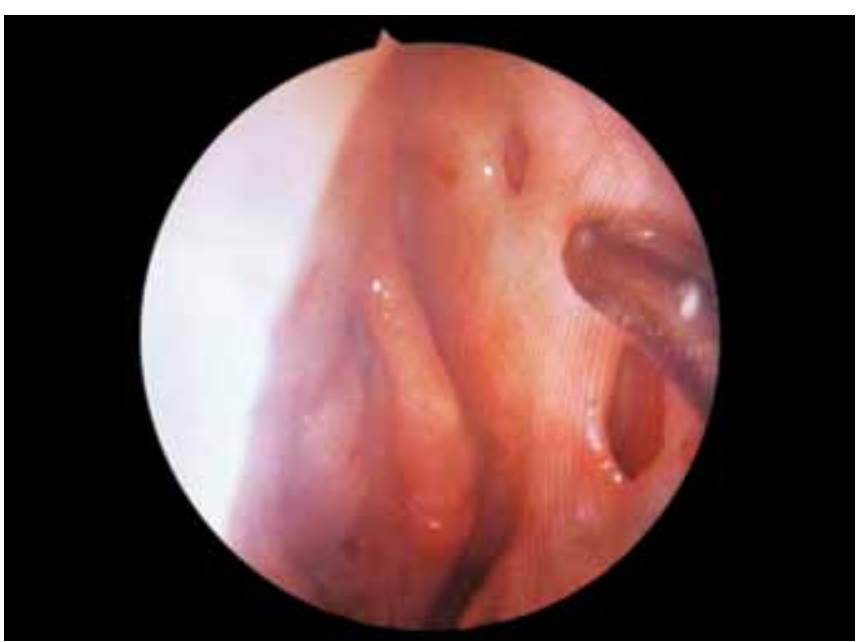

Fig. 3: Endoscopic picture after excision of the rhinoliths showing roomy nasal cavity, pushing of nasal septum to left with two septal perforations

a nasal mass mimicking malignancy or fungal ball. They appear as hard irregular masses which are dirty grayish brown or black in color. ${ }^{4}$

The reported complications include septal perforation, lateral wall and maxillary sinus erosion, oroantral or oronasal fistula. ${ }^{4,5,9}$

Differential diagnosis for rhinoliths are benign lesions like calcified nasal polyps, hemangiomas, ossifying fibromas, enchondromas and malignant tumors like osteosarcomas, chondrosarcomas. ${ }^{4,12}$ Apart from these, even chronic granulomatous diseases and osteomyelitis can also mimic rhinolith. $^{5}$

Computed tomography scan is an invaluable tool for diagnosis. ${ }^{12}$ The first radiological description of the rhinolith on CT scan was given by Maclintype in $1900 .{ }^{5}$ On CT they appear as a calcified high dense radiopaque mass with irregular border, which should be distinguished from other clinical entities such as ossifying fibroma, odontoma and osteoma or osteosarcoma. ${ }^{9,10,13}$

Treatment of rhinolith is by endoscopic removal..$^{9}$ But due to the long standing nature of these benign lesions, there is formation of granulation tissues and adhesions around the calcareous secretions. As a result, surgical removal may be difficult in such cases. In cases of larger rhinoliths, they can be crushed and broken down into smaller pieces thereby avoiding injury to the surrounding structures. Lithotripter has also been used for breaking the rhinolith. ${ }^{11}$ Concomitant treatment of associated conditions like sinusitis, septal deviation, perforation and oroantral fistula can be treated at the same time.

The report highlights one such rare case of two rhinoliths in a nasal cavity, presenting at an elderly age and mimicking fungal sinusitis or malignancy. The long standing rhinoliths had led to multiple septal perforations and pan sinusitis. 


\section{CONCLUSION}

Rhinolith, although rare should be considered in the differential diagnosis of unilateral nasal mass in the elderly. Most cases of rhinolith are accidentally detected while treating conditions like sinusitis. In contrast, our case presented with a nasal mass mimicking malignancy or a fungal ball.

Our report highlights one such rare case of two rhinoliths presenting as a nasal mass in an elderly male, which were removed.

\section{REFERENCES}

1. Davis $\mathrm{O}$, Wolff A. Rhinolithiasis and maxillary antrolithiasis. Ear, Nose Throat J 1985;64:421-426.

2. Polson CJ. On rhinoliths. J Laryngol Otology 1943;58(3):79-116.

3. The Rhinolith: a possible differential diagnosis of a unilateral nasal obstruction. Case Reports in Medicine 2010; Article ID 845671. doi: 10.1155/2010/845671.

4. Ozdemir S, Akbas G, Selçuk T, Sayar H, Tarkan O. An unusual copresentation of rhinolithiasis and squamous cell carcinoma in the nasal cavity. J Craniomaxillofac Surg 2012;40: 137-139.

5. Pitt SKJ, Routt PGJ. Rhinoliths presenting during routine radiography: two cases. Dent Update 2000;29:505-507.

6. Abdel Latif SM, Hady SA, Moustafe HM. Crystallographic study of rhinoliths. J Laryngol Otol 1979;93:1205-1209.

7. Bafageeh SA. Rhinolith. Pak J Otolaryngol 1993;9:90-93.

8. Bhatia JN, Gupta AK. Rhinolith. Indian J Otolaryngol HeadNeck Surg 1978;30:46.

9. Özkan O, Bingöl F, Budak A. The importance of endoscopy in the diagnosis and treatment of rhinolithiasis: a case report. J Acute Disease 2013:70-72.

10. Singh I, Gathwala G, Yadav SPS, Singh I. Rhinolith. Indian J Otolaryngol Head-Neck Surg 2003;55(4):243-245.

11. Hadi U, Ghossaini S, Zaytoun G. Rhinolithiasis: a forgotten entity. Otolaryngol Head-Neck Surg 2002;126:48-51.

12. Chatziavramidis A, Kondylidou-SidiraA, Stefanidis A, SoldatouS. Longstanding rhinolith leading to anatomical alterations of the ipsilateral inferior nasal meatus and turbinate. BMJ Case Rep 2010. Published online 19 November 2010, doi: 10:1136/ bcr.07.2010.3155.

13. Chen C, Hao S, Tsang NM, Lee KF. Nasopharyngeal lith. Otolaryngol Head-Neck Surg 2001;125:576-578. 